Thrane müssen, um sie auf Harzöl prüfen zu können, mit 90 proc. Alkobol ausgeschüttelt werden. Die abgesetzte klare, alkoholische Lösung kann dann auf Harzöl geprüft werden. Die Reaction wird am besten in der Weise angestellt, dass man die Schwefelsäure auf der Wand behutsam herunterfliessen lässt, damit sich die Flüssigkeiten nicht mischen. War Harzöl zugegen, so entsteht an der Berührungsstelle ein rother oder violettrother Ring. Hier habe ich auch gefunden, dass bei geringem Harzölgehait und lichten Thrawen die $\mathrm{Mo}^{-}$ rawsky'sche Reaction empfindlicher ist; sobald aber der zu prüfende Thran ganz dunkel ist und daher auch die alkoholische Lösung dunkelgelb erscheint, so lässt uns diese Reaction im Stiche, es bleibt daher nichts übrig, als die Holde'sche Reaction anzuwenden. Auch für dunkle Mineralöle, die meistens zur Schmierung verwendet werden, ist die Morawsky'sche Reaction nicht zu brauchen, da die mit Essigsäureanbydrid erhaltene Lösung schon an und für sich dunkel gefärbt ist, welche die von Harzöl erzengte schwach violettrothe Färbung verdeckt. Es ist noch zu bemerken, dass Harze, so Colophonium, Schellack, auch die Morawsky'sche Reaction geben, daher muss man sich von deren Abwesenheit überzeugen, wenn die Morawsky'sche Reaction angewendet wird. Es ist daher in solchem Falle das zu untersuchende Öl zu verseifen und im unverseifbaren Theile das Harzöl zu suchen.

Für dunkle Mineralöle ist es viel rathsamer, auch bei der Holde'schen Reaction Schwefelsäure von 1,53 spec. G. zu verwenden, da es viele Mineralöle gibt, die mit Sehwefelsäure von 1,624 spee. G. geschüttelt, an und für sich dunkelgelb gefärbt werden, die die Reaction nicht so scharf hervortreten lassen. Es ist wahr, dass bei solchen Mineralölen mit Schwefelsäure von 1,53 spec. G. auch eine geibe Lösung erhalten wird, welche aber bei Weitem nicht so ist, als mit der stärkeren Säure. Aus obigen Gründen halte ich die Reaction mit Schwefelsäure von 1,53 spec. G. für besser, um so mehr, da die ron mir untersuchten Harzöle die Reaction eben so scharf gaben, als mit Schwefelsäure von 1,624 spec. G.

Es bliebe noch die Reaction zu untersuchen, die syrupartige Phosphorsäure mit Thranen und Harzölen erzeugt, auf welche Holde in seiner Arbeit aufmerksam macht.

Schädler ${ }^{2}$ ) gibt an, dass Thran, mit syrupartiger Phosphorsäure 5: 1 gemischt, S. 117 eine rothe Färbung gibt, die später in's Dunkle übergeht und noch in Verdünnungen von $1 \%$ sicher angezeigt wird. Holde wies die Unrichtigkeit dieser Angabe nach, und er erhielt nicht rothe, sondern schmutzig braunrothe Färbungen, die auch nur nach längerem Schütteln eintraten. Ich kann nur noch hinzufügen, dass Thrane von unzweifelhafter Herkunft mit genau nach Schädler's Vorschrift dargestellter, syrupartiger Phosphorsäure keine, oder nur sehr schwachrothe Färbungen erzeugen, die an Intensität auch nach längerem Schütteln nicht zunehmen. Holde erhielt bei Harzölen mit syrupartiger Phosphorsïure orangerothe bis blutrothe Färbungen, hingegen gaben die ron mir untersuchten Harzöle verschiedene Färbungen, und zwar violettrothe bis braunrothe. Da die mit syrupartiger Phosphorsäure erzeugten Färbungen verschiedene sind, kann man auf diese Basis keine qualitative Reaction gründen, trotzdem habe ich die Reaction weiter verfolgt, um die Empfindlichkeitsgrenze bestimmen zu können. Es wurde Harzöl sowohl mit Rüböl als Mineralöl gemischt und mit diesen Mischungen die Reactionen angestellt. Das Resultat war, dass ein und dasselbe Harzöl sowohl in Mischungen mit Mineral- als fetten Ölen dieselbe Empfindlichkeitsgrenze besitzt, dass aber bei verschiedenen Harzölen auch diese verschieden ist; so konnte von einem Harzöl noch 5 Proc. nachgewiesen werden, während ein anderes bei demselben Harzölgehalt die Reaction nicht mehr gab. Ein anderes Harzöl gab noch bei 5 Proc. die Reaction, bei 3 Proc. konnte sie nicht mehr erhalten werden. Diese Reaction kann also sowohl an Empfindlichkeit wie an Charakteristik weder mit der Morawsky'schen oder Holde'schen verglichen werden. bahnen.

Budapest, Laboratorium d. K. ung. Staatseisen-

\section{Über die Entstehung der braunen Flecke und die Vermeidung derselben bei der Spiegelfabrikation.}

\section{Von}

\section{Dr. Adolf Jolles und E. Wild.}

Aus dem chemisch-mikroskopischen Laboratorium ron Dr. M. und Dr. Ad. Jolles in Wien.

Die erste Aufgabe, die wir uns gestellt hatten, war die genaue Feststellung der örtlichen Lage der braunen Flecke, d. h. ob dieselben am Spiegel oder am Spiegelbelage 
festsässen. Zu diesem Zwecke wurde der Spiegelbelag durch verdünnte Säuren vorsicbtig entfernt, wobei die braunen Flecke an der Oberfläche des Glases in Form eines feinen braunen Häutchens sichtbar wurden. Die Analyse dieser feinen braunen Schicht auf dem Glase ergab, dass dieselbe eine durchsichtige Haut ron Schwefelsilber sei, welche $z$ wischen Silberbelag und Glas fest an letzterem haftet.

Diese Thatsache führte uns zur Annahme, dass an der Oberfiäche des Glases eine Schwefelverbindung vorhanden war, welche mit dem metallischen Silber des Belages Schwefelsilber bildete, wodurch die braune Haut zum Vorschein kommen konnte.

Zu den Rohstoffen zur Herstellung von Spiegelglas gehört bekanntlich schwefelsaures Natrium. Wird ferner der Streckofen mit Steinkoble geheizt, so jst die Möglichkeit vorhanden, dass die Schwefligsäure, welche die Kohle liefert, auf dem Glase schwefelsaures Natrium bildet. Es kann deshalb auf gestreckten Tafeln schwefelsaures Natron, sowohl Spuren des ursprünglichen Zusatzes, als auch neugebildetes aus dem Schwefel der Kohle herstammendes, sich befinden. Während Neubildungen von schwefelsaurem Natron nur bei etwaiger oxydirender Flamme im Streckofen entstehen können, wird sich bei der in der Regel reducirenden Streckofenflamme aus schwefelsaurem Natron Schwefelnatrium bilden, welches später in $\mathrm{Be}$ rührung mit der Versilberungsflüssigkeit schwarze Flecke hervorrufen muss.

Von besonderer Wichtigkeit ist es, die Spiegelglastafeln sobald wie möglich von der Glashütte und von der Polieranstalt zur Versilberung kommen zu lassen, weil durch das Liegenlassen die Gläser stark bäutig werden, was für die Spiegelfabrikation von grossem Nachtheile wäre. Auf diese, dem Spiegelfabrikanten wohlbekannte Erscheinung möchten wir hinweisen, um erklären zu können, wieso es kommt, dass die braunen Flecke an den Spiegeln beim Versilbern nur dort auftreten, wo letztere während des 'Transportes zufällig nass geworden sind.

Das Häutigwerden beruht auf einer Einwirkung der feuchten, kohlensäurehaltigen Luft auf die Glasoberflächen, von welchen wir bereits nachgewiesen haben ${ }^{1}$ ), dass sie durch Betriebsfehler manchmal an Kalk ärmer, an Alkali und Schwefel reicher sind, wie das Glasinnere. In der erwähnten Abhandlung fanden wir neben anderen Schwefelverbindungen nur mikroskopische Krystalle von schwefelsaurem Natron, welche in der

i) Sprechs. 1890 No. 35; Fischer's Jabresb. 1890 S. 742.
Glasoberfläche eingeschmolzen waren und sich in dem Glase in einem Zustande beginnender Auflösung befanden.

Es ist bekannt, dass schwefelsaures Natron in geringen Mengen sich unzersetzt in der Glasmasse lösen kann. - Aber das von uns neuerdings nachgewiesene Schwefelnatrium ist im Glasflusse auch löslich.

Die winzige Menge des oberflächlich gelösten Schwefelnatriums wird das Glas allerdings nicht merkbar gelb färben, trotzdem man mit viel Schwefelnatrium Glas stark gelb färben kann. Allein es wird unter günstigen Bedingungen das Silber des Belages in schwarzes Schwefelsilber sich verwandeln können, und dieser Process wird überall dort, wo er sich nur im geringsten Maasse vollzieht, zu Bräunungen Anlass geben. So lange das Schwefelnatrium in der Glasmasse gelöst ist, ist es gewissermaassen durch die umhüllende Glasmasse unschädlich gemacht. Wenn aber eine solche Stelle in feuchtem oder nassem Zustande längere Zeit der Einwirkung der Luft ausgesetzt bleibt, dann wird die schützende Glasoberfläche zersetzt, es wittert kohlensaures Natron aus, die Tafel überzieht sich mit einer oft irisirenden farblosen Haut von opalartiger Kieselsäure und das gelöste Schwefelnatrium wird frei. Wahrscheinlich zersetzt sich auch dieses weiter, und unter dem Einfluss von Sauerstoff und Kohlensäure tritt Schwefelwasserstoff- und Schwefelausscheidung ein.

Es ist klar, dass durch Abwischen oder Abwaschen mit Wasser der in den feinsten Poren der Glasoberfläche sitzende Schwefel nicht weggebracht wird, wohl aber in einem alkalischen Versilberungsbad örtlich allmählich wieder in Schwefelnatrium verwandelt wird. Es sind also fortlaufende Zersetzungsprocesse an einmal nass gewordenen Tafeln die Ursache, dass sich an den ehemals nassen Stellen besonders schwefelreicher Tafeln beim Versilbern dunkle Schwefelsilberflecke bilden.

Eine weitere Aufgabe war es für uns, ein Mittel zu finden, um diese Flecke zu verbindern. $\mathrm{Zu}$ diesem Mittel führte uns folgender Versuch.

Die Spiegel wurden vorerst mit Alkohol behaudelt, um den Lack zu entfernen, daun wurde die Miniumschicht vorsichtig mit feuchter Watte weggewischt und die Silberschicht in verdünnter Salpetersäure gelöst. Nachdem der Belag vollkommen entfernt war, wurde das braune Häutchen im Glase deutlich sichtbar. Nach vielen Versuchen ist es uns gelungen, das braune Häutchen durch kochende Natronlauge wegzubringen, so dass nach 
kurzer Zeit von den Flecken nichts mehr zu sehen war. Statt Natronlauge kann auch Sodalösung verwendet werden, beide müssen 5 bis 10 proc. sein. Nach unseren Laboratoriumsversuchen genügt ein 1 Minute langes Kochen des Spiegelglases in 10 proc. Natronlauge, um den Fleck vollkommen zu entfernen.

Um nun zu verhindern, dass die Spiegelglastafeln beim Versilbern fleckig werden, ist es sehr empfehlenswerth, dieselben zuerst in 10 proc. Sodalösung 1 Minute lang zu kochen, mit Wasser sehr gut zu spülen und dann zu versilbern. Diese Behandlung kann, ohne dem Glanz der Glastafeln zu schaden und ohne ein Springen derselben befürchten zu müssen, dadurch ausgeführt werden, dass sie in einem Gitterkasten in die Sodalösung gestellt und diese allmählich auf $100^{\circ}$ erhitzt wird. Nennenswerthe Kosten werden dadurch nicht entstehen.

Unter allen Versuchen, die wir im Laboratorium ausgefübrt haben, ist die Behandlung der Spiegelscheiben mit Sodalösung die beste und empfehlenswertheste. Natronlauge ist im Stande, auch das schwerlösliche braune Schwefelsilber zu lösen. Wo aber dieser Bildung vorgebeugt werden soll, ist Sodalösung besser, weil es wohl Kieselsäure, Schwefel, Natriumsulfit u. s. w. löst, aber die eigentliche Glasfläche weit sicherer unangegriffen lässt, wie Natronlauge.

\section{Brennstoffe, Feuerungen.}

Vorrichtung zum Aufgeben von Brennstoff bei Schachtöfen. Nach F. Kawalewski und L. du Pasquier (D.R.P. No. 55801) setzt sich der Kanal $G$ (Fig. 127) im Gewölbe des Schachtofens in einen Fülltrichter $H$ fort, in dessen Achse eine Stange a verschiebbar angebracht ist, welche oben eine Handkurbel $b$ und unten einen den Kanal $G$ verschliessenden Kolben $b^{1}$ trägt. Diese Stange wird durch einen Ring $c$ gestützt, der mittels eines Gelenkes $d$ mit einem Handhebel $e$ verbunden ist, welcher an dem zur Führung der Stange $a$ dienenden Bock drehbar gelagert ist. Soll Brennstoff aufgegeben werden, so wird durch Handhabung des Hebels $e$ der Kolben $b^{1}$ gesenkt und dadurch dem im Kanal befindlichen Brennstoff der Zugang zum Schacht freigegeben. Zugleich wird durch Drehen des Kolbens der auf diesen niedergesunkene Brennstoff bis an die entfernteren Stellen

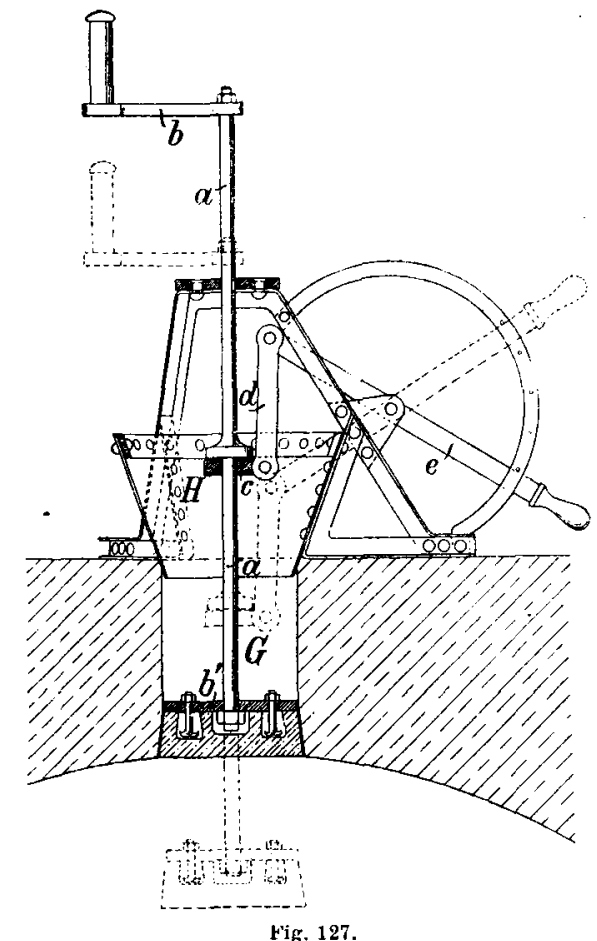

des Ofeneinsatzes geschleudert. Die Regelung erfolgt durch Heben und Senken des Kolbens in Verbindung mit schnellerem oder langsamerem Drehen desselben.

\section{Hüttenwesen.}

Beschickungsvorrichtung für Hochöfen u. dgl. Schachtöfen. Nach Solvay \& Co. (D.R.P.No.55811) wird der bekannte stillstehende Kegel ersetzt durch einen unter der Einschicköffnung $D$ (Fig. 128 und 129) um eine lothrechte Achse durch Getriebe $G$ drehbaren Kegel $A$, dessen unterer Rand eine Schneckenlinie bildet.

Will man z. B. ein gleichförmiges Beschicken in ebener Schichte erzielen, d. h. so, dass jeder Punkt des zu bedeckenden Querschnittes das gleiche Verhältniss an Materialien erhält, so wird die Kegelbasis durch eine der Gleichung $r=a \sqrt{e}$ entsprechende Spirale begrenzt, wobei $r$ den von 0 bis zu einer Länge gleich der des Ofenschachtradius oder einer geringeren Länge wachsenden Radiusvector und $\theta$ den zwischen diesem und dem Anfangsradius eingeschlossenen Winkel bedeutet. Jeder einem constanten Winkel im Centrum entsprechende Theil dieser Schneckenlinie erzeugt eine constante ringförmige Fläche. Die auf diesen Schneckenkegel $A$ fallende Beschickung vertheilt sich dessen Umfang gemäss, und wenn der Kegel während des Einfallens der Beschickung eine volle Umdrehung macht, so wird die Beschickung als ebene Schichte gleichmässig über den Schachtquerschnitt vertheilt. Schüttet man ferner hinter einander verschiedene Materialien ein und lässt man 